\title{
Piezoresistivity and Mechanical Behavior of Metal-polymer Composites under Uniaxial Pressure
}

\author{
Majid Kazemian Abyaneh ${ }^{1}$, Satish Ekar $^{2}$ \& Sulabha K. Kulkarni ${ }^{3}$ \\ ${ }^{1}$ Sincrotrone S.C.p.A., Area Science Park, SS-14, 163.5 Km, Basovizza-34149, Trieste, Italy \\ ${ }^{2}$ Department of Physics, University of Pune, Pune-411007, India \\ ${ }^{3}$ Indian Institute of Science Education and Research (IISER), Pune-411021, India \\ Correspondence: Majid Kazemian Abyaneh, Sincrotrone S.C.p.A., Area Science Park, SS-14, 163.5 Km, \\ Basovizza-34149, Trieste, Italy. Tel: 39-040-3758823. E-mail: majid.kazemian@elettra.trieste.it
}

Received: March 26, 2012

Accepted: April 5, 2012 Online Published: June 5, 2012

doi:10.5539/jmsr.v1n3p50

URL: http://dx.doi.org/10.5539/jmsr.v1n3p50

\begin{abstract}
The piezoresistive response of copper and nickel particles reinforced polydimethylsiloxane (PDMS) polymer, forming metal-polymer composites were investigated under uniaxial pressure. Electrical and mechanical properties of composite samples containing $\mathrm{Cu}$ and $\mathrm{Ni}$ conductive fillers at different loadings in polydimethylsiloxane (PDMS) polymer matrix have been presented here. Well-dispersed metal-polymer composites were fabricated by a wet mixing route. The resulting $\mathrm{Cu}-\mathrm{PDMS}$ and Ni-PDMS composite samples show the resistivity changes by more than 2 and 10 orders of magnitude around 4MPa pressure, respectively. The mechanical tests have shown that these composite samples have high stress sensitivity which makes them good piezoresistive candidates for practical applications.
\end{abstract}

Keywords: piezoresistivity, strain, composites, polymer film

\section{Introduction}

In the last few decades, composites based on conducting fillers incorporated in the insulator matrices have been a focus of research (Tognana et al., 2009; Canavese et al., 2012; Abyaneh et al., 2006, 2007). Different metal and semiconductor fillers reinforced into the insulators can enhance the electrical conductivity of the host materials are now widely used in circuit breakers, electromagnetic shielding, electrical heating, antistatic devices, etc. (Jeong et al., 2009; Al-saleh et al., 2009; Fosbury et al., 2003; Krupa et al., 2007). It is also possible that composites made of conductive fillers embedded in insulator host matrix undergo a transition from insulating to conductive regime by varying some chemical or physical parameters; like temperature changes, applied pressure, gas absorption, current or magnetic field (Heaney, 1996; Beruto et al., 2005; Chen et al., 2004; Bendark, 2002). Among the various composites, metal-polymer composites have attracted great interest. These composites have found a growing demand for their multifunctionality and application as sensors and offering cost effectiveness, flexibility, lightweight, etc. (Delmonte, 1989).

Some of the metal-polymer composites can be used as pressure sensors and their sensing ability is based on piezoresistivity defined as the response of electrical resistivity to an external deformation. Starting from an insulating state, by increasing the pressure on the samples, the adjacent particles in close proximity favor the tunneling process. Higher compression leads to the formation of more percolation paths through the samples and results into a conductor-like behavior.

In this paper, we report the fabrication of $\mathrm{Cu}$ and $\mathrm{Ni}$ particles reinforced into the PDMS polymer medium with a wet chemical route. Composite samples are made with different concentration and their piezoresistance behavior is investigated at room temperature under uniaxial pressure. The $\mathrm{Cu}$ and Ni-PDMS composite samples show the resistivity changes by more than 2 and 10 orders of magnitude around $4 \mathrm{MPa}$ pressure, respectively. The mechanical property of the samples is studied and their stress-strain curves are measured and the strain sensitivity of the composite samples is evaluated. It is shown that the Ni-PDMS samples have high strain sensitivity and are good candidates for practical applications. 


\section{Experimental}

\subsection{Materials and Samples Preparation}

The composite samples were prepared by mixing the filler and polymer in solution as the following (Figure 1(a)). All chemicals were used as they were received. The given amounts of copper or nickel particles (Thomas Baker; India, LR grade) were mixed with chloroform (Thomas Baker; India, AR grade) to wet the particles completely. The PDMS (Dow Corning, SYLGARD 184) consists of the "elastomer" (viscous liquid) and "hardener" (liquid). Elastomer and hardener are used in 10:1 weight ratio in these experiments. PDMS is an elastic polymer which is transparent and highly insulating $\left(\sim 1.2 \times 10^{14} \Omega\right.$-cm) with dielectric constant $\sim 2.65$. The elastomer was added to the copper or nickel solution and mixed thoroughly to form a homogeneous solution. The resulting solutions were heated to $\sim 60^{\circ} \mathrm{C}$ so that the solvent evaporated completely. When cooled down to room temperature, hardener was added to the viscous solution and degassed under vacuum for 1 hour. The heat curing of the mixture was made inside a Teflon mould at $80^{\circ} \mathrm{C}$ for $20 \mathrm{hrs}$. To obtain different concentration of copper or nickel, we simply change the initial amount of metal powders. The samples were later cut into $5 \times 5 \times 2 \mathrm{~mm}^{3}$ size for pressure-resistivity measurements, mechanical tests and other characterizations.

\subsection{Characterization and Instrumentation}

Scanning electron microscope (SEM) images and energy dispersive data were recorded using a JEOL JSM $6360 \mathrm{~A}$ microscope with $10 \mathrm{kV}$ operating voltage.

The DC electrical resistivity measurements of all the samples were carried out at room temperature using two-probe technique. The schematic diagram in Figure 1(b) shows the set-up used for piezoresistance measurements under uniaxial pressure. A Keithley 238 source measure unit was used for I-V measurements which provides a driven guard for output and sense probes to eliminate the effect of leakage current in high resistance measurements. The current range is $1 \mathrm{~A}$ to $10^{-4} \mathrm{nA}$ and voltage range is $110 \mathrm{~V}$ down to $100 \mu \mathrm{V}$. The current was kept fixed and applied in the direction parallel to the applied uniaxial pressure and voltages were measured at different applied pressures. The copper plates were used as electrodes and silver paste contacts were made on the samples to ensure a good electrical contact of the $\mathrm{Cu}$ plate with the samples. The uniaxial pressure was mechanically applied to the samples during the resistivity measurements using a homemade instrument and pressure was measured using a digital force gauge. The instrument consists of a sample holder, a coarse screw, a fine screw and a force gauge. The sample holder is placed on the gear box of the fine screw. Pressure gauge is attached to the coarse screw. The sample can be compressed between the course screw head and the sample plate holder. Fine pressure adjustment on the sample is effected using the fine screw.
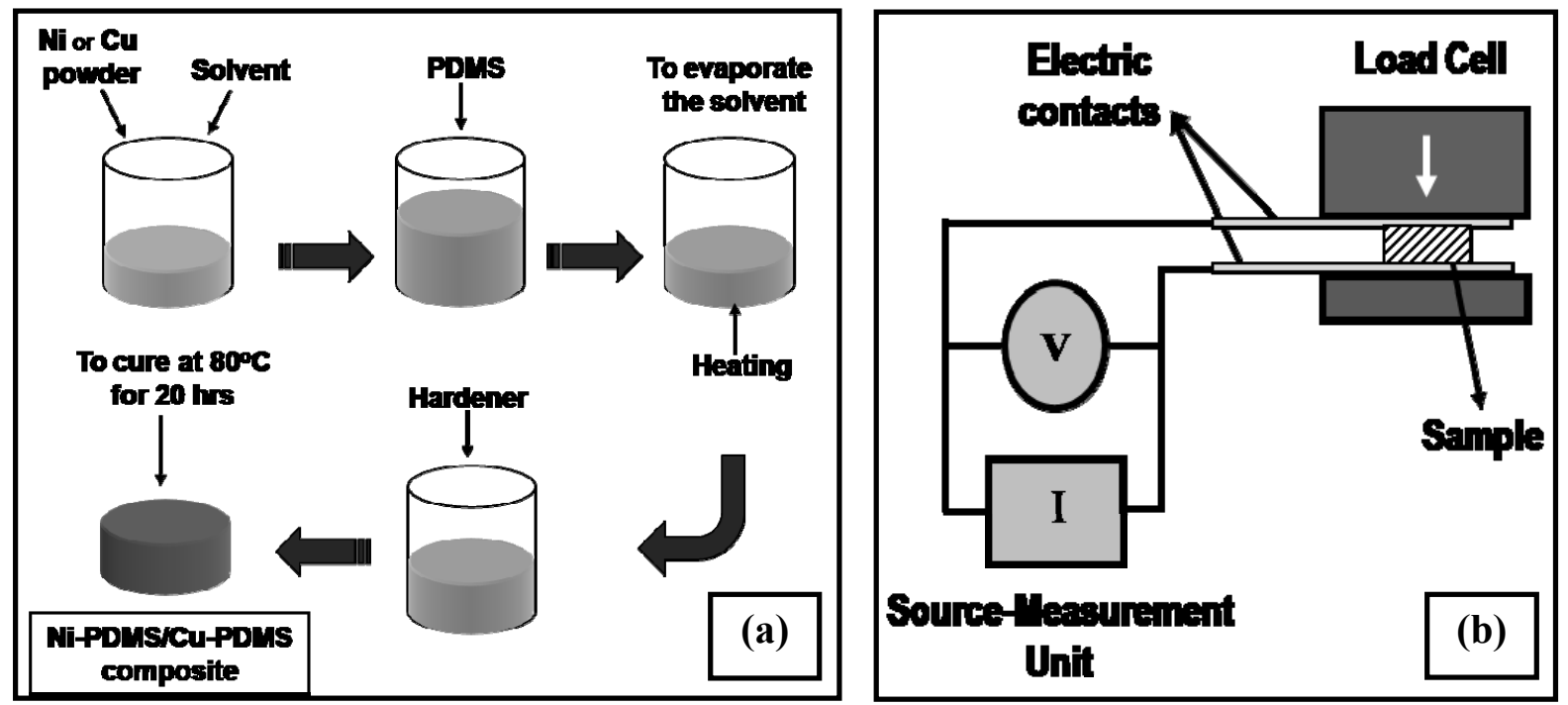

Figure 1. (a) Illustration for fabrication procedure of Ni/Cu-PDMS composites and (b) Scheme of the set-up used for resistance measurements under uniaxial compression 


\section{Results and Discussion}

\subsection{Electron Microscopy}

First, we look into the structure and morphology of the prepared metal-polymer composites. Figure 2(a) \& 2(b) show the scanning electron microscopy (SEM) images of 75.0\% Cu-PDMS and 83.0\% Ni-PDMS composite samples, respectively. The SEM images exhibit that the particles are well wrapped with PDMS polymer and dispersed properly in the medium. This is a direct result of the solution mixing method used for preparation of the composite samples. The size distribution of the metal particles in both composites is broad and covers the range of $\sim 0.1 \mu \mathrm{m}$ to $\sim 50 \mu \mathrm{m}$, but population of smaller particles in the Ni-PDMS composites is bigger than that of the $\mathrm{Cu}$-PDMS composites.
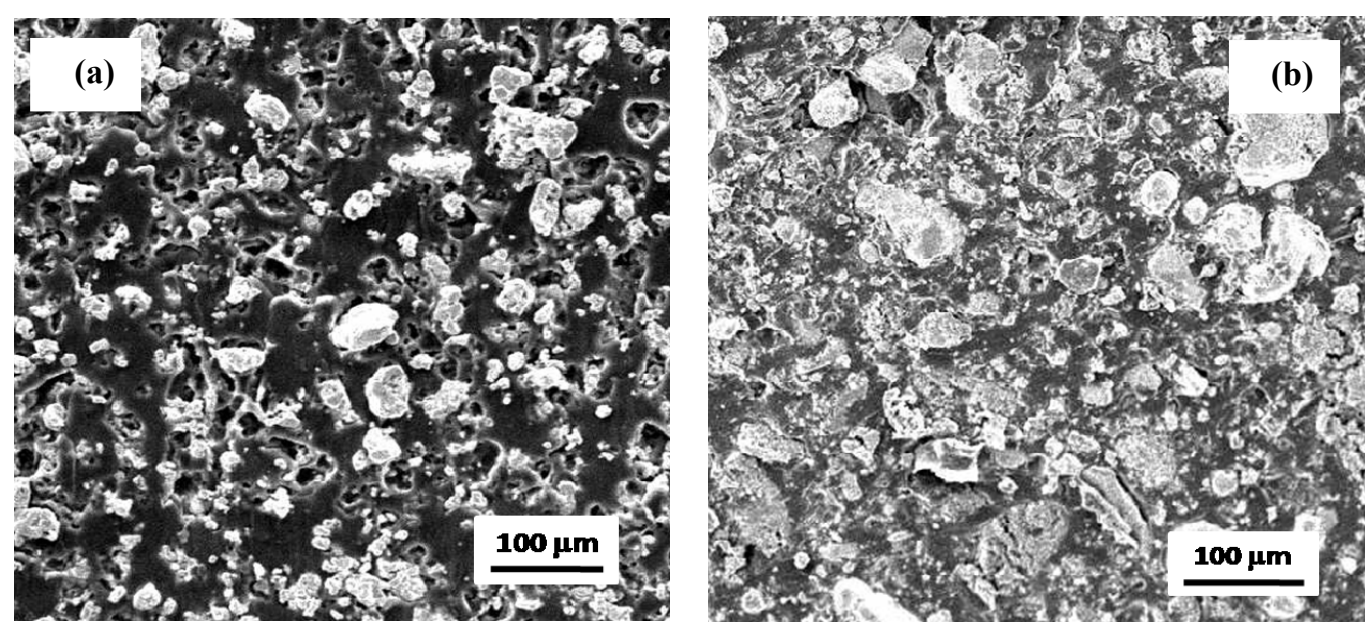

Figure 2. SEM images for (a) 75.0\% Cu-PDMS and (b) 83.0\% Ni-PDMS composite samples

\subsection{Piezoresistivity Measurements}

It is expected that by compressing the composites containing conductive fillers embedded in insulating matrix, the adjacent filler particles would come closer and the resistance would decrease. This is known as "piezoresistance effect". Various materials have been used as conductive fillers in some insulating polymers and elastomers, like carbon nanotubes, carbon black, graphite flakes and fibers, iron oxide particles etc. (Gau et al., 2009; Qu et al., 2007; Carmona et al., 1987; Hussain et al., 2001; Carlson et al., 2006; Ramajo et al., 2009). Many groups have reported the piezoresistivity of metal-polymer composites. Ausanio et al., 2006, have investigated the piezoresistivity of nickel- silicon type elastomer (Universal 5 or Essil 244) composites under uniaxial strain. They have reported that the composites can switch from insulating to conducting state accompanied by resistivity change of $\sim 9$ orders of magnitude. This can happen at pressure of $\sim 0.5$ or $1.5 \mathrm{MPa}$ with a little increment (2\%) of the relative deformation for different elastomers. Kchit et al., 2008, have studied the piezoresistivity of magnetorheological elastomers which are made by aligning nickel particles inside a silicon base polymer using a magnetic field before the curing process has started. They have shown seven orders of magnitude decrease from the initial resistivity $\left(\sim 10^{8} \Omega . \mathrm{cm}\right)$ under applied uniaxial pressure of around $0.3 \mathrm{MPa}$ for composites with $30 \%$ volume fraction. Bloor et al., 2005 and 2006 , have reported as large as $\sim 10^{14}$ fold decrease at $\sim 80 \%$ compression in the resistance of QTC ${ }^{\mathrm{TM}}$ samples made of nickel particles in some elastomer polymers using a patented route. They suggest that the extreme sensitivity of the resistance of QTC ${ }^{\mathrm{TM}}$ samples to any mechanical deformation is due to the sharp surface protrusions of the filler particles. Recently, we have shown that the resistance changes of approximately 7 orders of magnitude can be achieved in zinc-PDMS composites under the uniaxial pressure of $\sim 4 \mathrm{MPa}$ (Abyaneh et al., 2008). Mechanical behavior of the composites was investigated and it was shown that the Zn-PDMS composites exhibit reversible behavior even after many cycles of compression and decompression, indicating the excellent performance of Zn-PDMS composites as pressure sensors. We have made copper and nickel composites using solution mixing and investigated their electrical resistivity under uniaxial pressure. Figure 3(a) \& 3(b) show the resistivity versus applied uniaxial pressure for Cu-PDMS and Ni-PDMS composite samples with different filler concentrations, respectively. The resistivity for all uncompressed samples is around $10^{10} \Omega-\mathrm{cm}$ which is lower than of pure 
PDMS $\left(\sim 10^{14} \Omega\right.$-cm) but still falls in the insulating regime. This means that metal particles are sufficiently separated in the PDMS medium even for filler to polymer weight ratio up to $86.0 \%$. The insulator behavior of the uncompressed samples is due to the well rapped particles in the polymer matrix which prevents them from forming any conducting paths.
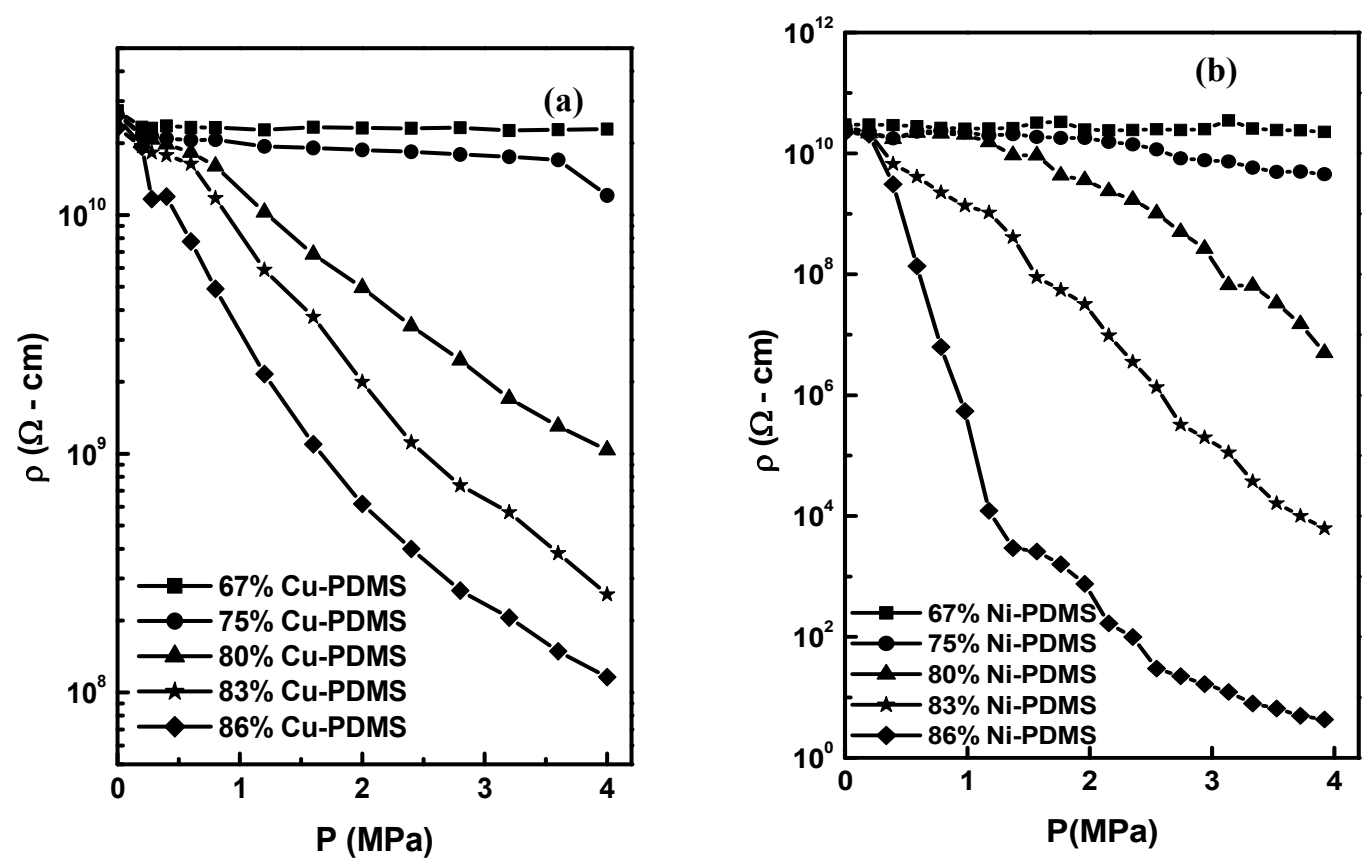

Figure 3. Resistivity of (a) Cu-PDMS and (b) Ni-PDMS composite samples respect to applied uniaxial pressure

As can be noticed from the figures, the samples lower than $75 \%$ filler to polymer weight ratio do not exhibit any changes in the resistivity with applied pressure. The applied pressure range, used in these experiments is $0-4$ $\mathrm{MPa}$ which is operating range of force gauge in our instrument. The $75 \% \mathrm{Cu}$-PDMS shows small change in resistivity at $\sim 4.0 \mathrm{MPa}$ and $75 \% \mathrm{Ni}$-PDMS start showing resistivity change at $\sim 2.0 \mathrm{MPa}$. The samples greater than $75 \%$ filler to polymer weight ratio starts showing more resistivity changes. As the weight percentage of metal particles is increased, the orders of magnitude changes in the resistivity have occurred. The resistivity of $86.0 \% \mathrm{Cu}$-PDMS decreased by $\sim 2$ orders of magnitude and reached minimum to $1.1 \times 10^{8} \Omega$-cm (The resistivity of pure copper is $\sim 1.7 \times 10^{-6} \Omega$-cm). The maximum change in resistivity occurs for $86.0 \%$ Ni-PDMS sample that decreased by $\sim 10$ orders of magnitude over a narrow uniaxial pressure range and reached to $0.4 \times 10^{1} \Omega$-cm (The resistivity of pure nickel is $\left.\sim 7 \times 10^{-6} \Omega-\mathrm{cm}\right)$. Such a small change in the resistivity for Cu-PDMS samples with respect to the Ni-PDMS samples with the same filler concentration, may be due to the thick oxide layer on the copper particles. It is shown that at room temperature, the oxidation of Nickel (Braaten et al., 1988) proceeds at a much slower rate than Copper (Platzman et al., 2008). Oxidation rate of both metals can be approximated by a logarithmic growth. When the oxide layer reaches a critical thickness, the oxidation rate attenuates due to the limiting of the electron tunnel current through the oxide film. The critical oxide layer thickness for $\mathrm{Ni}$ is at least 10 times smaller than $\mathrm{Cu}$ which can be estimated by comparing the experimental results for formation of oxide layers obtained by Braaten et al. (1988) and Platzman et al. (2008) using XPS measurements. We also have been measured the I-V curve for the composite samples at different compression. Uncompressed and highly compressed composites show ohmic behavior while they show highly nonlinear behavior in the intermediate region. The nonlinear behavior at the transition region appears as exponential decrease in resistivity graphs. The exponential behavior of the resistivity versus uniaxial pressure indicates that major conduction mechanism is that of tunneling (Abyaneh et al., 2008; Dawson et al., 1996).

\subsection{Mechanical Properties}

In the following, we have investigated the mechanical properties of cu-PDMS and Ni-PDMS composites which may play the main roles in their practical application (Martin et al., 2007; Xia et al., 2006). Figure 4(a) \& 4(b) show the stress-strain curves for cu-PDMS and Ni-PDMS composites with different metal to polymer 
concentrations, respectively. The Young's moduli have been determined from the slope of the plot of stress versus strain in the linear region. From these curves, one may easily observe that by increasing the metal powder concentration in PDMS polymer, the slopes of the curves have been increased. In other words, the lower concentrated composites are softer than the higher thereby giving rise to larger deflections for a given load.
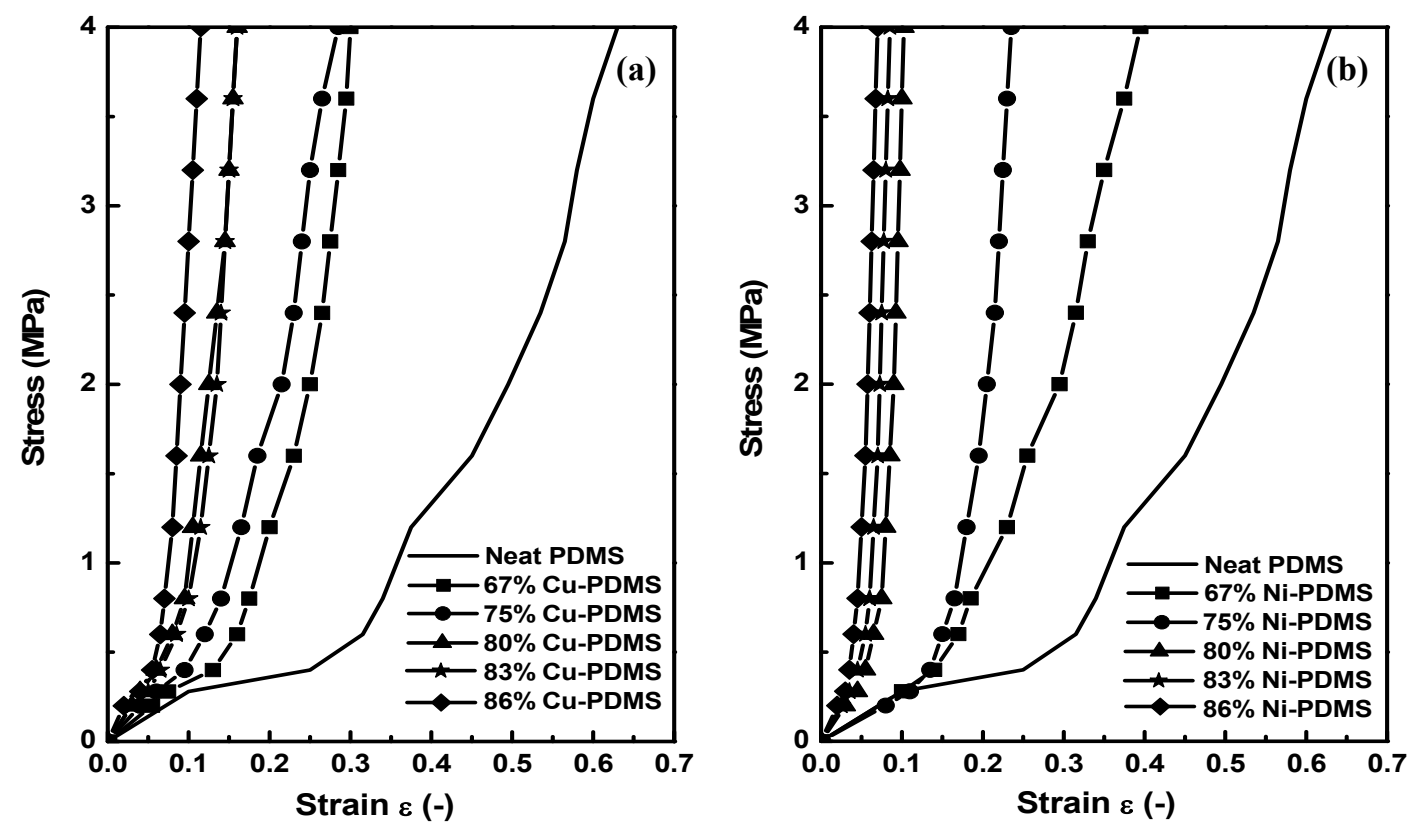

Figure 4. Stress-strain curves for (a) Cu-PDMS and (b) Ni-PDMS composite samples. Stress-strain curves for pure PDMS is included in both figures for comparison

The fractional change in resistance per unit applied strain called the gauge factor $(\Gamma)$, exhibits the strain sensitivity of a piezoresistive material and is a figure of merit for sensors. More strain sensitive materials have larger gauge factor. The gauge factor is defined as (Morten et al., 1979):

$$
\Gamma=\frac{d R}{R} \frac{1}{d \varepsilon}
$$

where $R$ is electrical resistance and $\varepsilon$ is strain. If we consider an isotropic film and the uniaxial compression applies along the thickness of the sample parallel to the current then we can write (Abyaneh et al., 2008),

$$
\Gamma=-G(1-2 v)-(1+2 v)
$$

where $G=\frac{1}{\rho} \frac{\partial \rho}{\partial \varepsilon_{\kappa}}$ for $\kappa \in[x, y, z]$, is the piezoresistivity coefficient and $v$ is the Poisson's ratio of the sample. The piezoresistivity coefficient can be determined from the gradient of graphs in which the logarithm of resistivity is plotted as a function of strain. Figure 5(a) \& 5(b) show the plot of $\ln \rho$ versus uniaxial compression (negative strain) for various concentrations of Cu-PDMS and Ni-PDMS composites, respectively. The fitted linear lines are shown with solid lines.

The gradient varied from -45 to -85 for Cu-PDMS with different concentration and the value of gauge factor is very low for any practical application. The slope for all Ni-PDMS curves is $|G| \cong 600$. Using Equation (2) and considering Poisson's ratio with value of $\sim 0.35$ for polymer composites we obtain $\Gamma \cong 180$, which is higher than that reported for QTC ${ }^{\mathrm{TM}}$ materials ( 105) (Bloor et al., 2006) and comparable to Au-coated Ni-epoxy composites ( 200) (Martin et al., 2003). The gauge factor of Ni-PDMS is a characteristic parameter for this composite and does not change by concentration. 

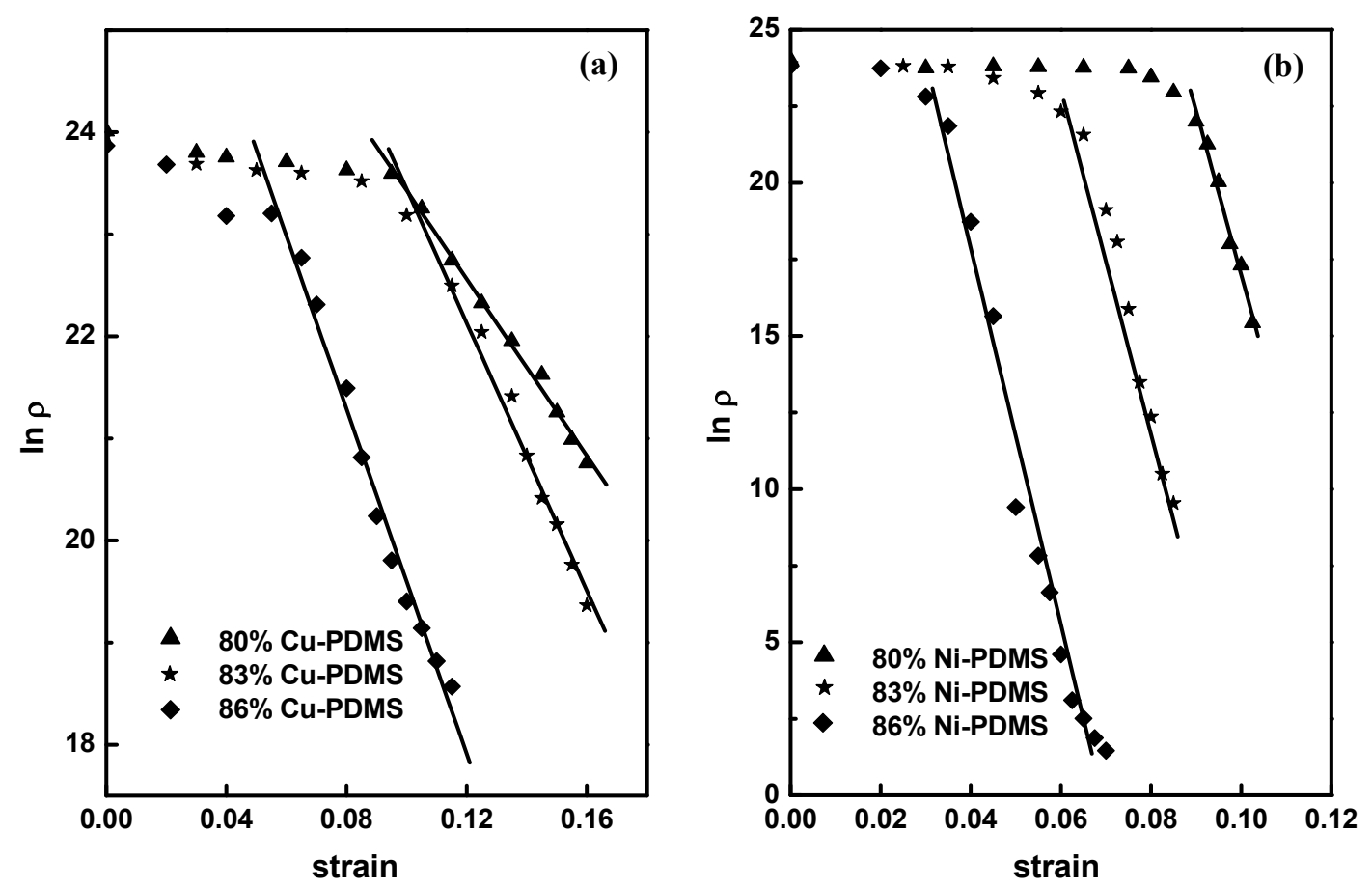

Figure 5. The Logarithm of resistivity versus the strain for (a) Cu-PDMS and (b) Ni-PDMS composite samples.

The solid lines are fitted linear lines

There are many theoretical equations which are proposed for the evolution of elastic modulus of the composites as a function of filler volume fraction. The simplest one is the Einstein's Equation (3) which is valid for dilute spherical particles in a Newtonian fluid with perfect adhesion between phases (Einstein, 1905).

$$
E_{c}=E_{m}\left(1+2.5 \phi_{f}\right)
$$

where $E_{c}$ and $E_{m}$ are Young's modulii of composite material and of the polymer matrix, respectively. $\phi_{f}$ is volume fraction of filler. The Thomas Equation (4) is a semi-theoretical equation that is based on monodispersed fillers and is valid for concentrated composites (Thomas, 1965).

$$
E_{c}=E_{m}\left[1+2.5 \phi_{f}+10.05 \phi_{f}^{2}+0.00273 \exp \left(16.6 \phi_{f}\right)\right]
$$

The Quemada Equation (5) is on the basis of particles' interactions and their different morphologies (Quemada, 1977).

$$
E_{c}=E_{m}\left(1-0.5 K \phi_{f}\right)^{-2}
$$

$K$ is a variable coefficient. Mooney has proposed Equation (6) which is for concentrated composites.

$$
E_{c}=E_{m} \exp \left[2.5 \phi_{f} /\left(1-L \phi_{f}\right)\right]
$$

The constant $\mathrm{L}$ is defined as crowding factor and predicted to be between 1.35 and 1.91 for the closed packing of monodisperse particles (Mooney, 1951). These equations are plotted in Figure 6 along with experimental data for Ni-PDMS composite samples. It seems that the Mooney equation is more close to experimental data. The Mooney's equation is an extension of the Enstein's equation applied for finite concentration and polydisperse system. The expected discrepancies are mainly due to the fact that the filler particles are not spherical and do not have perfect adhesion with their surrounding matrix. 


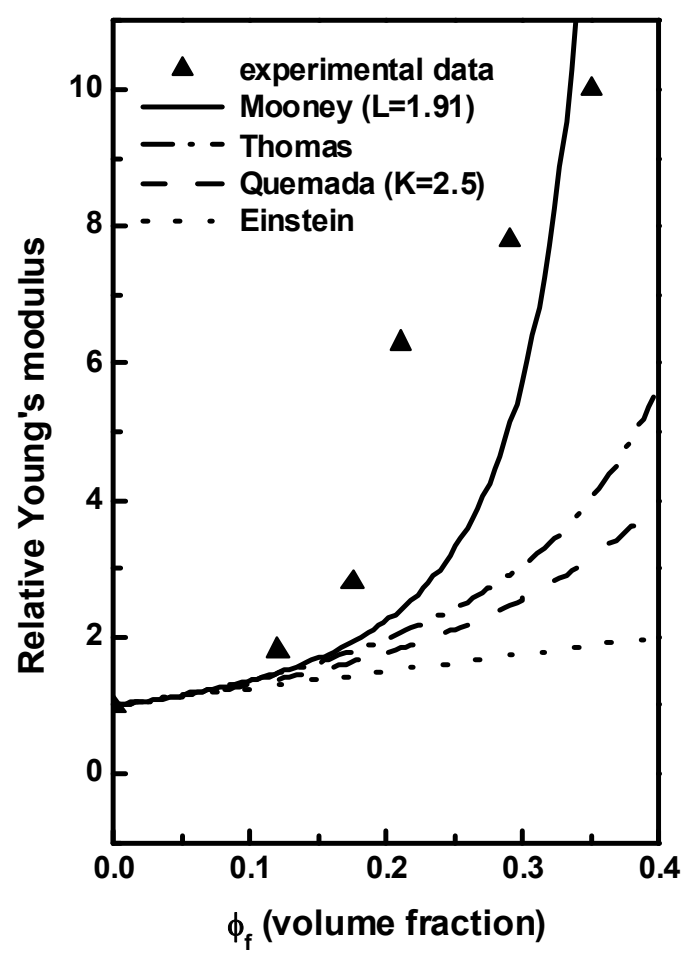

Figure 6. Relative Young's modulus versus volume fraction of filler for Ni-PDMS composite samples and some models given by Equations (3) to (6)

\section{Conclusions}

In summary, Cu-PDMS and Ni-PDMS composite samples were fabricated by mixing the metal filler and nonconducting polymer using a wet chemical method. The synthesis process can be easily extended to the screen printing or some other thick film deposition for batch synthesis. The piezoresistive behavior of the composite samples was investigated under uniaxial pressure at room temperature. The higher metal concentration of Ni-PDMS composites shows the resistivity changes by $\sim 10$ orders of magnitude. Undeformed and highly compressed composites show ohmic behavior while they show highly nonlinear behavior in the intermediate region. An extremely large gauge factor of 180 has been found for Ni-PDMS composites independent of the concentration of the fillers. Thus the performance of Ni-PDMS composites as pressure sensors is excellent and can find potential applications as flexible tactile sensors with large-area area in various field like robotics, biomechanics, etc. It is necessary to investigate the effect of size and shape along with size dependent mechanical effects on pressure dependent resistivity.

\section{Acknowledgments}

This work has been funded by DST and ISRO, India. S.K.Kulkarni acknowledges the support by UGC, India. The authors would like to thank Mr. V.V.Mujumdar for the fabrication of the pressure measurement unit.

\section{References}

Abyaneh, M. K., \& Kulkarni, S. K. (2008). Giant piezoresistive response in zinc-polydimethylsiloxane composites under uniaxial pressure. Journal of Physics D: Applied Physics, 41, 135405. http://dx.doi.org/10.1088/0022-3727/41/13/135405

Abyaneh, M. K., Paramanik, D., Varma, S., Gosavi, S. W., \& Kulkarni, S. K. (2007). Formation of gold nanoparticles in polymethylmethacrylate by UV irradiation. Journal of Physics D: Applied Physics, 40, 3771-3779. http://dx.doi.org/10.1088/0022-3727/40/12/032

Abyaneh, M. K., Pasricha, R., Gosavi, S. W., \& Kulkarni, S. K. (2006). Thermally assisted semiconductor-like to insulator transition in gold-poly(methyl methacrylate) nanocomposites. Nanotechnology, 17, 4129-4134. http://dx.doi.org/10.1088/0957-4484/17/16/023 
Al-saleh, M.H., \& Sundararaj, U. (2009). Electromagnetic interference shielding mechanisms of CNT/polymer composites. Carbon, 47, 1738-1746. http://dx.doi.org/10.1016/j.carbon.2009.02.030

Ausanio, G., Barone, A. C., Campana, C., Iannotti, V., Luponio, C., Pepe, G. P., \& Lanotte, L. (2006). Giant resistivity change induced by strain in a composite of conducting particles in an elastomer matrix. Sensors and Actuators A, 127, 56-62. http://dx.doi.org/10.1016/j.sna.2005.12.002

Bendark, S. (2002). Changes of thickness and self-excited oscillations of the conducting porous magnetoelast nearby percolation threshold caused by current flow. Materials Science and Engineering B, 94, 89-94. http://www.sciencedirect.com/science/article/pii/S092151070200082X

Beruto, D.T., Capurro, M., \& Marro, G. (2005). Piezoresistance behavior of silicone-graphite composites in the proximity of the electric percolation threshold. Sensors and Actuators A, 117, 301-308. http://dx.doi.org/10.1016/j.sna.2004.06.027

Bloor, D., Donnelly, K., Hands, P. J., Laughlin, P., \& Lussey, D. (2005). A metal-polymer composite with unusual properties. Journal of Physics D: Applied Physics, 38, 2851-2860. http://dx.doi.org/10.1088/0022-3727/38/16/018

Bloor, D., Graham, A., William, E. J., Laughlin, P., \& Lussey, D. (2006). Metal-polymer composite with nanostructured filler particles and amplified physical properties. Applied Physics Letters, 88, 102103. http://link.aip.org/link/doi/10.1063/1.2183359?ver=pdfcov

Braaten, N. A., Raaen, S., \& Grepstad, J. K. (1988). Oxidation of transition metal-rare earth interfaces: an XPS study. Journal of Physica Scripta, 37, 778-781. http://dx.doi.org/10.1088/0031-8949/37/5/020

Canavese, G., Stassi, S., Stralla, M., Bignardi, C., \& Pirri, C. F. (2012). Stretchable and conformable metal-polymer piezoresistive hybrid system. Sensors and Actuators A: Physical, http://dx.doi.org/10.1016/j.sna.2012.01.037

Carlson, W., Bartkowski, S., Schulze, W., \& Pilgrim, S. (2006). Testing of Piezoresistive Polyurethane-Fe $\mathrm{O}_{3}$ Composites. Ferroelectrics, 331, 83-88. http://dx.doi.org/10.1080/00150190600735234

Carmona, F., Canet, R., \& Delhaes, P. (1987). Piezoresistivity of heterogeneous solids. Journal of Applied Physics, 61, 2550-2557. http://link.aip.org/link/doi/10.1063/1.337932

Chen, S. G., Hu, J. W., Zhange, M. Q., Li, W. M., \& Rong, M. Z. (2004). Gas sensitivity of carbon black/waterborne polyurethane composites. Carbon, 42, 645-651. http://dx.doi.org/10.1016/j.carbon.2004.01.002

Dawson, J. C., \& Adkins, C. J. (1996). Conduction mechanisms in carbon-loaded composites. Journal of Physics: Condensed Matter, 8, 8321-8338. http://dx.doi.org/10.1088/0953-8984/8/43/024

Delmonte, J. (1989). Metal/Polymer Composites. London: Springer.

Einstein, A. (1905). Annalen der Physik, 17, 549-560. http://dx.doi.org/10.1002/andp.19053220806

Fosbury, A., Wang, S., Pin, Y. F., \& Chung, D. D. L. (2003). The interlaminar interface of a carbon fiber polymer-matrix composite as a resistance heating element. Composites: Part A, 34, 933-940. http://dx.doi.org/10.1016/S1359-835X(03)00208-2

Gau, C., Ko, H. S., \& Chen, H. T. (2009). Piezoresistive characteristics of MWNT nanocomposites and fabrication $\begin{array}{lllll}\text { as a polymer pressure sensor. Nanotechnology, } & \text { 20, } & 185503 .\end{array}$ http://dx.doi.org/10.1088/0957-4484/20/18/185503

Heaney, M. B. (1996). Resistance-expansion-temperature behavior of a disordered conductor-insulator composite. Applied Physics Letters, 69, 2602-2604. http://link.aip.org/link/doi/10.1063/1.117713

Hussain, M., Choa, Y. H., \& Niihara, K. (2001). Fabrication process and electrical behavior of novel pressure-sensitive composites. Composites: Part A, 32, 1689-1696. http://dx.doi.org/10.1016/S1359-835X(01)00035-5

Jeong, D. Y., Ryu, J., Lim, Y. S., Dong, S., \& Park, D. S. (2009). Piezoresistive $\mathrm{TiB}_{2} /$ silicone rubber composites for circuit breakers. Sensors and Actuators A, 149, 246-250. http://dx.doi.org/10.1016/j.sna.2008.11.022

Kchit, N., \& Bossis, G. (2008). Piezoresistivity of magnetorheological elastomers. Journal of Physics: Condensed Matter, 20, 204136. http://dx.doi.org/10.1088/0953-8984/20/20/204136 
Krupa, I., Mikova, G., Novak, I., Janigova, I., Nogellova, Z., Lednicky, F., \& Prokes, J. (2007). Electrically conductive composites of polyethylene filled with polyamide particles coated with silver. European Polymer Journal, 43, 2401-2413. http://dx.doi.org/10.1016/j.eurpolymj.2007.03.033

Martin, J. E., Anderson, R. A., Odinek, J., Adolf, D., \& Williamson, J. (2003). Observations of giant thermoresistance, piezoresistance, and chemiresistance: Controlling percolation in field-structured particle composites. Physical Review B, 67, 094207 (11pp). http://dx.doi.org/ 10.1103/PhysRevB.67.094207

Martin, M., Hanagud, S., \& Thadhani, N. N. (2007). Mechanical behavior of nickel + aluminum powder-reinforced epoxy composites. Materials Science and Engineering A, 443, 209-218. http://dx.doi.org/10.1016/j.msea.2006.08.106

Mooney, M. (1951). The viscosity of a concentrated suspension of spherical particles. Journal of Colloidal Science, 6, 162-170. http://dx.doi.org/10.1016/0095-8522(51)90036-0

Morten, B., Pirozzi, L., Prudenziati, M., \& Taroni, A. (1979). Strain sensitivity in film and cermet resistors: measured and physical quantities. Journal of Physics D: Applied Physics, 12, L51-L54. http://dx.doi.org/10.1088/0022-3727/12/5/003

Platzman, I., Brener, R., Haick, H., \& Tannenbaum, R. (2008). Oxidation of polycrystalline copper thin films at ambient conditions. Journal of Physics Chemistry C, 112, 1101-1108. http://dx.doi.org/10.1021/jp076981k

Qu, S., \& Wong, S. C. (2007). Piezoresistive behavior of polymer reinforced by expanded graphite. Composite Science and Technology, 67, 231-237. http://dx.doi.org/10.1016/j.compscitech.2006.08.008

Quemada, D. (1977). Rheology of concentrated disperse systems and minimum energy dissipation principle: I. Viscosity-concentration relationship. Rheologica Acta, 16, 82-94. http://dx.doi.org/10.1007/BF01516932

Ramajo, L. A., Cristobal, A. A., Botta, P. M., Porto Lopez, J. M., Reboredo, M. M., \& Castro, M. S. (2009). Dielectric and magnetic response of $\mathrm{Fe}_{3} \mathrm{O}_{4} /$ epoxy composites. Composites: Part A, 40, 388-393. http://dx.doi.org/10.1016/j.compositesa.2008.12.017

Thomas, D. G. (1965). Transport characteristics of suspension: VIII. A note on the viscosity of Newtonian suspensions of uniform spherical particles. Journal of Colloidal Science, 20, 267-277. http://dx.doi.org/10.1016/0095-8522(65)90016-4

Tognana, S., Salgueiro, W., Somoza, A., Pomarico, J. A., \& Ranea-Sandoval, H. F. (2009). Influence of the filler content on the thermal expansion behavior of an epoxy matrix particulate composite. Materials Science and Engineering B, 157, 26-31. http://dx.doi.org/10.1016/j.mseb.2008.12.003

Xia, X., Xie, C., Cai, S., Wen, F., Zhu, C., \& Yang, X. (2006). Effect of the loading and size of copper particles on the mechanical properties of novel $\mathrm{Cu} / \mathrm{LDPE}$ composites for use in intrauterine devices. Materials Science and Engineering A, 429, 329-333. http://dx.doi.org/10.1016/j.msea.2006.05.045 